\title{
Prevalence of DSM-IV disorders in a population-based sample of 5- to 8-year-old children: the impact of impairment criteria
}

\author{
Jolien Rijlaarsdam • Gonneke W. J. M. Stevens • \\ Jan van der Ende • Albert Hofman • Vincent W. V. Jaddoe • \\ Frank C. Verhulst $\cdot$ Henning Tiemeier
}

Received: 10 June 2014 / Accepted: 24 January 2015 / Published online: 26 February 2015

(C) The Author(s) 2015. This article is published with open access at Springerlink.com

\begin{abstract}
This study determined the impact of impairment criteria on the prevalence and patterns of comorbidity of child DSM-IV disorders. The validity of these impairment criteria was tested against different measures of mental health care referral and utilization. We interviewed parents of 1,154 children aged 5-8 years in-depth using the Diagnostic Interview Schedule for Children in Rotterdam, the Netherlands, to establish DSM-IV diagnosis. These children were randomly selected or oversampled based on Child Behavior Checklist ratings from a large populationbased study $(N=6,172)$. Referral data were extracted from the psychiatric interview as well as from a follow-up questionnaire. The results showed an overall prevalence of
\end{abstract}

J. Rijlaarsdam · V. W. V. Jaddoe

The Generation R Study Group, Erasmus MC-University Medical Center Rotterdam, Rotterdam, The Netherlands

J. Rijlaarsdam · J. van der Ende · F. C. Verhulst · H. Tiemeier $(\bowtie)$ Department of Child and Adolescent Psychiatry, Erasmus MCUniversity Medical Center Rotterdam, Rotterdam,

The Netherlands

e-mail: h.tiemeier@erasmusmc.nl

G. W. J. M. Stevens

Interdisciplinary Social Sciences, Faculty of Social Sciences,

University of Utrecht, Utrecht, The Netherlands

A. Hofman · V. W. V. Jaddoe $\cdot$ H. Tiemeier

Department of Epidemiology, Erasmus MC-University Medical

Center Rotterdam, Rotterdam, The Netherlands

V. W. V. Jaddoe

Department of Paediatrics, Erasmus MC-University Medical

Center Rotterdam, Rotterdam, The Netherlands

H. Tiemeier

Department of Psychiatry, Erasmus MC-University Medical

Center Rotterdam, Rotterdam, The Netherlands
DSM-IV disorders of $31.1 \%$ when impairment was not considered. This rate declined to $22.9 \%$ when mild impairment was required and declined even further, to $10.3 \%$, for more severe levels of impairment. Similarly, the overall comorbidity rate declined from 8.5 to 6.7 and $2.7 \%$ when mild and severe impairment were required, respectively. Virtually all children who attained symptom thresholds for a specific disorder, and had been referred to a mental health care professional because of the associated symptoms, also had mild impairment. The requirement of severe impairment criteria significantly increased diagnostic thresholds, but for most disorders, this definition captured only half of the clinically referred cases. In conclusion, prevalence was highly dependent upon the criteria used to define impairment. If severe impairment is made a diagnostic requirement, many children with psychiatric symptoms and mild impairment seeking mental health care will be undiagnosed and possibly untreated.

Keywords Child psychiatric disorders - Prevalence . Comorbidity $\cdot$ Impairment $\cdot$ Referral for treatment

\section{Introduction}

Childhood psychiatric disorders often interfere with social and academic functioning and show considerable continuity over time [1-3]. Well-planned interventions targeting children with psychiatric disorders may serve not only to ameliorate current problems and associated impairment in functioning but also to prevent the persistence of problems into adolescence and adulthood. Epidemiological research investigating the prevalence of psychiatric disorders and patterns of comorbidity may inform nosology and intervention planning. 
Despite advances in the DSM's [4] operational definitions of disorder and psychiatric epidemiological methods, the differentiation of developmentally normal from abnormal behaviors and emotions has proven challenging [5-11]. For example, worries about losing major attachment figures or fear of dogs are common in young children and may be inappropriately diagnosed as psychiatric symptoms or disorders (e.g., separation anxiety or specific phobia). Perhaps most strikingly, structured lay interviews in epidemiological surveys have revealed unexpectedly high rates of psychiatric disorders in young children, particularly phobias $[7,8,10]$. A prime alteration in DSM-IV from DSM-III was the requirement of clinically significant distress or impairment in social, occupational, or other important areas of functioning for psychiatric diagnosis [4]. In the recently published DSM-5 [12], the criterion of clinically significant impairment or distress in functioning is retained.

While the phenomenology and symptoms of disorders were operationally defined in DSM based on expert panel consensus, impairment was left open to judgment by clinicians [5]. Despite the widespread acceptance that the requirement of clinically significant impairment helps in the differentiation from normality, there is a lack of an agreed operational definition in epidemiological studies [7, 10]. Hence, efforts to approximate the clinical diagnostic process are hampered. Specifically, there has been poor agreement between the several measures of clinical significant impairment, which has been partially attributed to the choice of thresholds (e.g., "some" or mild versus "a lot" or severe) [13]. For example, impairment questions with dichotomous ("a lot" versus "not a lot") and scaled ("a lot," "some," "a little," or "not at all") response options have been used interchangeably [14]. It is unclear whether respondents answering "some" to the scaled question would answer "a lot" or "not a lot" to the dichotomous question [14].

Such differences ("some" versus "a lot") in the operationalization of the DSM impairment criterion may have substantial effects on prevalence. Whereas some studies found the rate of childhood oppositional defiant disorder (ODD) to decline by approximately $38 \%$ (from 2.9 to $1.8 \%$ ) [15], others found this rate to decline by $24 \%$ (from 6.6 to $5.0 \%$ ) [16] and $10 \%$ (from 13.4 to $12.1 \%$ ) [17], or to remain at the same level (8.4\%) [18]. Similarly, studies of adolescents have demonstrated substantial [19] and little [20] or no declines [21] in rates of ODD when impairment was included in the diagnostic criteria. Inconsistent patterns of findings in previous research may be explained by differences in methodologies and the lack of an accepted operational definition of the DSM impairment criterion. However, few have reported effects of multiple operationalizations of impairment on the prevalence rates of individual childhood disorders.
This study determined the 3-month prevalence and patterns of comorbidity of DSM-IV disorders in a large population-based cohort of children aged 5-8 years. We examined the impact of multiple definitions of impairment on the prevalence and comorbidity of disorders. In addition, we tested the validity of these definitions of impairment against different measures of mental health care referral and utilization. We expected prevalence to be highly dependent upon the criteria used to define impairment, with more significant decreases in rates for more severe levels of impairment. Furthermore, we expected that the addition of impairment to the diagnostic criteria serves not only as a way to increase thresholds, but may also more accurately indicate clinically referred cases.

\section{Method}

Sample and procedure

This research was embedded in the Generation R Study, a population-based cohort from fetal life onward. Sample ascertainment and participation have been described in detail elsewhere [22]. Pregnant women living in the study area in Rotterdam, the Netherlands, with an expected delivery date between April 2002 and January 2006 were invited to participate. In this paper, we utilize data obtained as part of a Generation R early school age (5-9 years) follow-up study [22]. The study was conducted in accordance with the guidelines proposed in the World Medical Association Declaration of Helsinki and was approved by the Medical Ethical Committee of the Erasmus University Medical Center, Rotterdam. Written consent was obtained from all participants.

In the Generation R Study, a total of 9,276 children were invited for follow-up assessments at early school age, and 8,305 of these children participated. For the purpose of efficient estimation of prevalence rates of psychiatric disorders, we focused on a subsample enriched for psychopathology and employed a two-stage design. We defined cutoff points based on the Child Behavior Checklist preschool form (CBCL 1.5/5) [23] total problems score (the top 15 percent) and the syndrome scale scores (the top 2 percent) to identify children with a high probability of disorder. The psychometric properties of the CBCL are well established [23].

The CBCL preschool form (age 1.5-5) as well as the CBCL school form (age 6-18) could have been applied to children in the age range of 5-7 years. Because we anticipated the majority of children (59\% in this sample) to be younger than 6 years at the time of assessment, and assessment waves relied on sending the parents of children the same questionnaire, we decided to use the CBCL preschool 
form for all children. CBCL data were available for 6,252 children, $75 \%$ of those eligible $(N=8,305)$ and $67 \%$ of those in the original cohort $(N=9,276)$. Non-respondents in the original cohort $(N=3,024)$ did not differ from respondents $(N=6,252)$ in terms of gender. However, non-respondents were less often Dutch (39 versus $61 \%$, $\left.\chi^{2}(1)=369.26, p<0.001\right)$ and their parents were more often single ( 29 versus $\left.14 \%, \chi^{2}(1)=16.67, p<0.001\right)$ and lower educated $\left(\chi^{2}(2)=51.93, p<0.001\right)$. We excluded 80 children aged $\geq 8$ years, leaving a sample of 6,172 children (mean age $=6.03, \mathrm{SD}=0.40$ ) eligible for in-depth diagnostic assessment.

Given our focus on a subsample of children enriched for psychopathology, all children scoring above the predetermined cut-off points (screen-positives, $N=1,080$ ), and a random selection of children scoring below the cut-off points (screen-negatives, $N=330$ ) were recruited for indepth interview. These 330 selected screen-negative children did not differ from the non-selected screen-negative children in terms of child gender, national origin, parental marital status, and maternal educational level (all $p>0.05$ ). Parents of the total of 1,410 targeted children were contacted by telephone to make an appointment for a home visit. If parents were not reached after at least eight attempts, contact was made by mail or by personal visits. No informant could be reached for 102 of these 1,410 children. Interview data were obtained from 1,166 participants, $89 \%$ of those reached. Non-respondents $(N=142)$ did not differ from respondents $(N=1,166)$ in terms of gender and parental marital status. However, non-respondents were less often Dutch (45 versus $55 \%$, $\left.\chi^{2}(1)=4.65, p=0.031\right)$ and their mothers were lower educated $\left(\chi^{2}(2)=8.42, p=0.015\right)$. Twelve children were older than 9 years at the time of interview and were excluded from analyses. This left 1,154 children with interview data (mean age $=6.74, \mathrm{SD}=0.59$ ) in our full sample $(N=6,172)$.

\section{Psychiatric interview}

The diagnostic interview schedule for children-young child version (DISC-YC) [24] is a highly structured DSM-IV [4]-based interview to be administered to the parents or caregivers of children aged 3-8 years. The DISC-YC is a developmentally appropriate adaptation of the DISC-parent version (DISC-P; intended for children aged 6-17 years). Building on the well-validated DISC-P with only minor developmentally specific adaptations including the timeframe (generally the past 3 months instead of the past year) and question wording (e.g., "other children" instead of "other people"), the DISC-YC was nevertheless subjected to psychometric testing. Data on the acceptable to high overall reliability of the DISC-YC symptom scales have not been formally published but were obtained through personal communication with the authors and were also reported by others (test-retest reliability for attentiondeficit/hyperactivity disorder (ADHD) scales $=0.67$, for $\mathrm{ODD}=0.88$, for anxiety and depression scales $=0.57$ 0.81 ) [17]. The timeframe of the DISC-YC for determining the presence of disorders is generally the past 3 months. Only for dysthymia and conduct disorder a 1-year timeframe was used.

We used the computer-assisted DISC-YC, which is programmed to derive DSM-IV diagnoses by applying the algorithms provided by the developers. The DISC-P had previously been translated into Dutch and verified by back translation into English. Two research staff members translated the parts of the DISC-YC that had been adapted to be developmentally appropriate. Subsequently, a third research staff member involved in the DISC-P translation reviewed and approved their translations.

Like the DISC-P, the DISC-YC is designed to be administered by lay interviewers. Six interviewers were trained to administer the DISC-YC during an official training program held by the DISC-YC staff. Bilingual interviewers (Dutch and Turkish, Dutch and Arabic or Dutch and Amazigh (Berber)) were trained to interview parents who were not sufficiently fluent in Dutch to participate. Although the interviewers were informed of the study purposes, they were blind to the CBCL groupings (scores below or above cut-off points).

Impairment. At the end of each diagnostic section in the DISC-YC, there are 6-stem/contingent question pairs to assess "impairment" from the symptoms of the disorder. These DISC-YC questions on impairment include whether the symptoms reported (1) caused caretakers to be annoyed or upset, (2) prevented the child from doing things or going places with family, (3) prevented the child from doing things or going places with other children, (4) caused problems with activities, tasks, or play, (5) caused teachers or additional caretakers to be annoyed or upset, and (6) caused distress to the child. These impairments are rated on a three-point scale: "a lot of the time," "some of the time," or "hardly ever" (or in the case of problems with activities: "very bad," "bad," or "not too bad").

The current study reports prevalence rates at three levels: (1) "all children meeting the DSM-IV symptom criteria" including all children displaying the minimum number of symptoms needed for diagnosis, (2) "at least mild impairment" imposing the requirement that at least one impairment has been given an "intermediate" or "severe" rating (i.e., "some of the time," "a lot of the time," "bad," or "very bad"), and (3) "severe impairment" imposing the requirement that at least one impairment has been given a "severe" rating (i.e., "a lot of the time" or "very bad"). 
The multiple definitions of impairment were first validated against the DISC-YC items on mental health care referral and utilization. At the end of each diagnostic section in the DISC-YC, parents were asked whether or not the child had been to someone at a hospital, a clinic or other mental health institute in the last three months because of the symptoms. The impairment definitions were then validated against referral data collected in the whole Generation $\mathrm{R}$ cohort as part of a subsequent assessment wave at age 8 years $(N=4,399$ in the full sample; $N=795$ in the DISC-YC sample). Parents rated whether or not the child had been referred to an in- or out-patient clinic in the last year as a result of anxiety, mood, ADHD, or disruptive behavior problems.

\section{Statistical analysis}

Statistical analyses were carried out using the computer package SPSS Complex Samples Statistics (IBM SPSS Statistics version 20.0). This procedure takes account of the complex survey design and applies weights to adjust for unequal sampling probabilities to represent rates of disorder in the full sample $(N=6,172)$. Specifically, we restricted the analyses to those 1,154 children with interview data and assigned a sampling weight to each child, representing the inverse of the stage two sampling fraction (i.e., screen-positives in stage one/screen-positives in stage two; screen-negatives in stage one/screen-negatives in stage two) [25].

The 3-month prevalence of the DISC-YC-derived diagnoses was determined without and with the additional impairment criteria. Logistic regression analysis was used to examine the associations of child gender with child psychiatric disorder and the odds of comorbidity between pairs of diagnoses. When low rates of individual diagnoses precluded meaningful logistic regression analysis, DSM-IV diagnoses were collapsed into the following diagnostic groupings [19]: anxiety, mood, ADHD, and conduct/oppositional disorder. Comorbidity between ODD and conduct disorder could not occur because, according to the DSM-IV criteria, a diagnosis of conduct disorder takes precedence over a diagnosis of ODD.

Validity testing of the impairment criteria was performed in two steps. We first used the referral data extracted from the DISC-YC and examined the extent to which children who attained symptom thresholds for a specific disorder, and had been referred as a result of the symptoms, also had mild or severe impairment. We then used the referral data collected as part of the subsequent data wave at age 8 years. We examined the weighted prevalence of referral to an in- or out-patient clinic at age 8 years per DISC-based diagnostic grouping and per level of impairment.

\section{Results}

\section{Prevalence of DSM-IV disorders}

Table 1 reports the 3-month prevalence of DSM-IV disorders in selected 5- to 8-year-old children $(N=1,154)$ weighted to represent rates in the full sample $(N=6,172)$. Rates of disorder are presented according to level of impairment required for diagnosis. The children who had data on the DISC-YC at age 6 years (i.e., weighted subsample) were representative of the full sample in terms of gender (52 versus $50 \%$ were boys, respectively), national origin (64 versus $62 \%$ were Dutch, respectively), maternal education (10 versus $12 \%$ attained $\leq 3$ years general secondary school, respectively), and parental marital status (10\% were single).

Of the children in this sample, $31.1 \%$ attained DSM-IV symptom thresholds for any disorder. The two most prevalent disorders were ODD (10.5\%) and specific phobia (10.7\%), followed by ADHD (8.7\%). Within the ADHD category, the rates for the inattentive type, the hyperactive type, and the combined type were $3.2,2.8$, and $2.6 \%$, respectively. The overall prevalence of $31.1 \%$ declined to $22.9 \%$ when mild impairment was required and declined even further, to $10.3 \%$, for more severe levels of impairment. We compared prevalence rates and their $84 \%$ confidence intervals to detect change due to a more stringent case definition (see also Table 1) [26]. Whereas rates of behavioral disorders and mood disorders remained largely unchanged when mild impairment was added to the diagnostic criteria, rates of anxiety disorders, and in particular specific phobia, declined considerably. When severe impairment was required, prevalence rates significantly declined for both anxiety (from 12.7 to $2.4 \%$ ) and behavioral (from 16.4 to $8.1 \%$ ) disorders.

Table 2 shows prevalence estimates for DSM-IV diagnostic groupings by child gender. As shown in Table 2, none of the associations with child gender reached statistical significance in logistic regression analysis. However, a marginally significant gender difference was found in the occurrence of any disorder without the requirement of impairment (35.3\% in boys versus $26.5 \%$ in girls, $\mathrm{OR}=0.66,95 \% \mathrm{CI}=0.44-1.00)$.

Impairment

We first validated the impairment criteria against DISCbased, diagnosis-specific referral rates. All children (or all except one for social phobia, generalized anxiety, ADHD, and conduct disorder) who attained symptom thresholds for a specific disorder, and had visited a mental health care professional because of the associated symptoms, also had mild impairment. In contrast, approximately half of the 
Table 1 Three-month prevalence of DSM-IV disorders among 5- to 8-yearolds according to level of impairment required for diagnosis $(N=6,172)$

Weighted prevalence estimates (95\% confidence intervals) based on Diagnostic Interview Schedule for Children (DISC) interviews in 1,154 selected children to represent rates in the full population-based sample $(\mathrm{N}=6,172)$. The timeframe of the DISC for determining the presence of disorders was generally the past 3 months. Only for dysthymia and conduct disorder a 1-year timeframe was used

$A D H D$ attention-deficit/ hyperactivity disorder

a Decrease in rate is statistically significant: mild impairment required versus impairment not required

b Decrease in rate is statistically significant: severe impairment required versus impairment not required

c Decrease in rate is statistically significant: severe impairment versus mild impairment required

\begin{tabular}{|c|c|c|c|c|c|c|}
\hline \multirow[t]{2}{*}{ Diagnosis } & \multicolumn{2}{|c|}{$\begin{array}{l}\text { Impairment not } \\
\text { required }\end{array}$} & \multicolumn{2}{|c|}{$\begin{array}{l}\text { Mild impairment } \\
\text { required }\end{array}$} & \multicolumn{2}{|c|}{$\begin{array}{l}\text { Severe impairment } \\
\text { required }\end{array}$} \\
\hline & $\%$ & $(95 \% \mathrm{CI})$ & $\%$ & $(95 \% \mathrm{CI})$ & $\%$ & $(95 \% \mathrm{CI})$ \\
\hline Any disorder & 31.1 & $(26.9-35.6)$ & $22.9^{\mathrm{a}}$ & $(19.3-26.9)$ & $10.3^{\mathrm{b}, \mathrm{c}}$ & $(7.9-13.2)$ \\
\hline \multicolumn{7}{|l|}{ Anxiety disorders } \\
\hline Any anxiety disorder & 12.7 & $(9.9-16.1)$ & $7.8^{\mathrm{a}}$ & $(5.7-10.7)$ & $2.4^{\mathrm{b}, \mathrm{c}}$ & $(1.4-4.1)$ \\
\hline Social phobia & 1.4 & $(0.7-2.5)$ & 1.2 & $(0.6-2.4)$ & 0.6 & $(0.2-1.6)$ \\
\hline Separation anxiety & 1.3 & $(0.6-2.8)$ & 0.9 & $(0.4-2.2)$ & $0.3^{\mathrm{b}, \mathrm{c}}$ & $(0.1-0.5)$ \\
\hline Specific phobia & 10.7 & $(8.1-14.0)$ & $6.1^{\mathrm{a}}$ & $(4.1-8.8)$ & $1.6^{\mathrm{b}, \mathrm{c}}$ & $(0.8-3.3)$ \\
\hline Generalized anxiety disorder & 0.5 & $(0.3-0.7)$ & 0.4 & $(0.3-0.7)$ & $0.1^{\mathrm{b}, \mathrm{c}}$ & $(0.0-0.3)$ \\
\hline Obsessive-compulsive disorder & 0.5 & $(0.1-1.6)$ & 0.2 & $(0.1-0.3)$ & 0.1 & $(0.0-0.2)$ \\
\hline Post-traumatic stress disorder & 0.1 & $(0.0-0.2)$ & 0.1 & $(0.0-0.2)$ & 0.0 & $(0.0-0.2)$ \\
\hline \multicolumn{7}{|l|}{ Mood disorders } \\
\hline Any mood disorder & 0.8 & $(0.3-2.2)$ & 0.8 & $(0.3-2.2)$ & 0.4 & $(0.1-1.6)$ \\
\hline Major depressive episode & 0.4 & $(0.1-1.8)$ & 0.4 & $(0.1-1.8)$ & 0.3 & $(0.1-1.9)$ \\
\hline Dysthymia & 0.5 & $(0.1-1.6)$ & 0.4 & $(0.1-1.7)$ & 0.1 & $(0.0-0.3)$ \\
\hline \multicolumn{7}{|l|}{ Behavioral disorders } \\
\hline Any behavioral disorder & 16.4 & $(13.4-19.9)$ & 15.5 & $(12.6-18.9)$ & $8.1^{\mathrm{b}, \mathrm{c}}$ & $(6.1-10.7)$ \\
\hline Any ADHD & 8.7 & $(6.6-11.3)$ & 8.0 & $(6.1-10.6)$ & $4.6^{\mathrm{b}, \mathrm{c}}$ & $(3.2-6.7)$ \\
\hline ADHD-inattention & 3.2 & $(2.0-5.2)$ & 2.7 & $(1.6-4.6)$ & 1.4 & $(0.7-2.9)$ \\
\hline ADHD-hyperactive & 2.8 & $(1.8-4.5)$ & 2.7 & $(1.7-4.4)$ & 1.6 & $(0.8-3.0)$ \\
\hline ADHD-combined & 2.6 & $(1.7-4.1)$ & 2.6 & $(1.7-4.1)$ & 1.7 & $(0.9-3.1)$ \\
\hline Oppositional defiant disorder & 10.5 & $(8.1-13.5)$ & 10.1 & $(7.8-13.1)$ & $4.6^{\mathrm{b}, \mathrm{c}}$ & $(3.2-6.7)$ \\
\hline Conduct disorder & 0.3 & $(0.2-0.5)$ & 0.2 & $(0.1-0.4)$ & 0.1 & $(0.0-0.3)$ \\
\hline \multicolumn{7}{|l|}{ Miscellaneous } \\
\hline Nocturnal enuresis & 6.6 & $(4.6-9.2)$ & $2.2^{\mathrm{a}}$ & $(1.2-3.9)$ & $0.2^{\mathrm{b}, \mathrm{c}}$ & $(0.1-0.4)$ \\
\hline Diurnal enuresis & 0.7 & $(0.3-1.7)$ & 0.6 & $(0.2-1.6)$ & $0.1^{\mathrm{b}, \mathrm{c}}$ & $(0.0-0.2)$ \\
\hline Encopresis & 1.5 & $(0.7-3.2)$ & 1.2 & $(0.5-2.7)$ & 0.7 & $(0.2-2.2)$ \\
\hline Tourette's disorder & 0.0 & $(0.0-0.2)$ & 0.0 & $(0.0-0.2)$ & 0.0 & $(0.0-0.2)$ \\
\hline
\end{tabular}

children who attained symptom thresholds and had visited a mental health care professional because of symptoms associated with social phobia, generalized anxiety, ADHD and ODD, also had severe impairment. Only for separation anxiety, all children who attained symptom thresholds for disorder, and had been referred for treatment, also had severe impairment.

Next, we examined the weighted prevalence of referral at age 8 years per DISC-based diagnostic grouping and per level of impairment. Table 3 shows that for all diagnostic groupings the weighted prevalence of referral was consistently low in children falling below DISC symptom thresholds for diagnosis (range $=1.3-2.2 \%$ ). As expected, these rates of referral were higher in those children who attained symptom thresholds for mood (44\%), ADHD (26.8\%), and conduct/oppositional (15.1\%) disorders. Furthermore, these rates of referral were higher in those children who attained symptom thresholds with mild (range $=15.3-$ $45.5 \%$ ) and severe (range $=24.0-82.0 \%$ ) impairment. Only few children attaining symptom thresholds for anxiety disorders, with or without the requirement of impairment, were referred (e.g., rate of referral was $4.9 \%$ when severe impairment was required).

\section{Comorbidity of DSM-IV disorders}

The prevalence of general comorbidity (i.e., two or more DSM-IV disorders of any type) was $8.5 \%$ when impairment was not considered, and decreased to 6.7 and $2.7 \%$ when mild and severe impairment criteria were employed, respectively. The odds of comorbidity between pairs of diagnoses were examined using logistic regression analysis. Table 4 shows that when impairment was not considered, ADHD was associated with anxiety $(\mathrm{OR}=2.62$, $95 \% \mathrm{CI}=1.36-5.07)$ and conduct/oppositional disorders $(\mathrm{OR}=5.97,95 \% \mathrm{CI}=3.15-11.3)$. When mild impairment was required, the associations between disorders were generally large and statistically significant, with the exception that mood disorders were unrelated to all other disorders (see Table 4). That is, ADHD was associated with conduct/ 
Table 2 Three-month prevalence estimates for DSM-IV diagnostic groupings according to level of impairment required for diagnosis and gender

\begin{tabular}{lccc}
\hline Diagnostic grouping & Boys $(N=637) \%(95 \% \mathrm{CI})$ & Girls $(N=517) \%(95 \% \mathrm{CI})$ & OR $(95 \% \mathrm{CI})$ \\
\hline Any disorder, impairment not required & $35.3(29.3-41.7)$ & $26.5(20.9-33.0)$ & $0.66(0.44-1.00)$ \\
Any disorder with mild impairment & $25.6(20.5-31.4)$ & $19.9(15.1-25.8)$ & $0.72(0.46-1.12)$ \\
Any disorder with severe impairment & $10.7(7.7-14.7)$ & $9.7(6.5-14.4)$ & $0.90(0.51-1.59)$ \\
Anxiety, impairment not required & $12.4(8.8-17.3)$ & $13.0(9.0-18.3)$ & $1.05(0.60-1.83)$ \\
Anxiety with mild impairment & $8.2(5.4-12.3)$ & $7.5(4.6-11.9)$ & $0.90(0.46-1.79)$ \\
Anxiety with severe impairment & $3.3(1.7-6.4)$ & $1.4(0.6-3.4)$ & $0.41(0.13-1.28)$ \\
Mood, impairment not required & $0.8(0.2-3.1)$ & $0.8(0.2-3.6)$ & $0.94(0.12-7.17)$ \\
Mood with mild impairment & $0.8(0.2-3.2)$ & $0.8(0.2-3.6)$ & $0.99(0.12-7.86)$ \\
Mood with severe impairment & $0.2(0.1-0.5)$ & $7.0(4.4-10.9)$ & $3.45(0.50-24.04)$ \\
ADHD, impairment not required & $10.3(7.4-14.1)$ & $6.8(4.2-10.7)$ & $0.65(0.36-1.20)$ \\
ADHD with mild impairment & $9.2(6.5-12.9)$ & $4.3(2.3-7.9)$ & $0.72(0.38-1.34)$ \\
ADHD with severe impairment & $4.9(3.1-7.8)$ & $8.4(5.5-12.8)$ & $0.86(0.38-1.95)$ \\
Conduct/oppositional, impairment not required & $12.9(9.4-17.5)$ & $8.3(5.4-12.6)$ & $0.62(0.35-1.12)$ \\
Conduct/oppositional with mild impairment & $12.2(8.8-16.6)$ & $3.9(2.1-7.2)$ & $0.65(0.36-1.18)$ \\
Conduct/oppositional with severe impairment & $5.5(3.5-8.7)$ & $0.69(0.31-1.55)$ \\
\hline
\end{tabular}

Weighted prevalence estimates (95\% confidence intervals) based on Diagnostic Interview Schedule for Children (DISC) interviews in 1,154 selected 5- to 8-year-olds to represent rates in the full population-based sample $(N=6,172)$

$A D H D$ attention-deficit/hyperactivity disorder

oppositional disorder $(\mathrm{OR}=6.44,95 \% \mathrm{CI}=3.32-12.5)$ and anxiety disorders $(\mathrm{OR}=4.17,95 \% \mathrm{CI}=1.94-8.97)$. Furthermore, anxiety disorders were associated with conduct/oppositional disorder $(\mathrm{OR}=2.78(95 \% \mathrm{CI}=1.28$ 6.01). We additionally examined the association between disorders imposing a requirement of severe impairment. Again, ADHD was associated with conduct/oppositional disorder $(\mathrm{OR}=9.69,95 \% \mathrm{CI}=3.80-24.7)$ and anxiety disorders $(\mathrm{OR}=7.19,95 \% \mathrm{CI}=1.98-26.13)$. Also, anxiety disorders were associated with conduct/oppositional disorder $(\mathrm{OR}=7.12,95 \% \mathrm{CI}=1.95-26.0)$ and mood disorder $(\mathrm{OR}=10.28,95 \% \mathrm{CI}=1.41-74.68)$.

\section{Discussion}

In this population-based cohort, an estimated 31.1 and $22.9 \%$ of 5 - to 8-year-old children had at least one psychiatric disorder without and with the additional requirement of impairment, respectively. This rate declined to $10.3 \%$ when more severe levels of impairment were required for diagnosis. The prevalence of general comorbidity was $8.5 \%$ when impairment was not considered, and declined to 6.7 and $2.7 \%$ when mild and severe impairment were required for diagnosis, respectively.

The overall prevalence of psychiatric disorders with mild impairment is consistent with previous reports of young, school-age children [16, 18, 27]. The most common disorders were behavioral disorders, particularly ODD.
Behavioral disorders without the consideration of impairment occurred in $16.4 \%$ of our sample, which resembled the $15.9 \%$ observed by McArdle et al. [16] and the $14.3 \%$ observed by Carter et al. [18], who also investigated the impact of impairment criteria on rates of disorder [16, 18]. Whereas McArdle et al. [16] found the rate of behavioral disorders to decline by $34 \%$ (to $10.5 \%$ ) when moderate impairment was required for diagnosis, the current study and the Carter et al. [18] study found this rate to remain largely unaltered.

The lack of a clear pattern in the impact of impairment criteria may be attributable to the considerable variation in the operationalizations or measurements of functional impairment. For example, McArdle et al. [16] used a measure of global impairment, whereas the current study and the Carter et al. [18] study used DISC-based diagnosis-specific impairment measures. There has been poor agreement between these two measures of impairment, which has been partially attributed to the choice of thresholds (e.g., mild or moderate versus "a lot" or severe) [13]. However, few studies have reported effects of multiple operationalizations of impairment on the prevalence of childhood disorders. We showed that the requirement that the symptoms interfered with children's functioning "a lot of the time" (conceptualized here as severe impairment) had a significantly greater impact on the rates of anxiety and behavioral disorders than the requirement that the symptoms interfered with children's functioning "some of the time" (conceptualized here as 
Table 3 Prevalence of mental health care referral and utilization at age 8 years per DSM-IV diagnostic grouping and level of impairment $(N=6,172)$

\begin{tabular}{ll}
\hline Diagnostic groupings & $\begin{array}{l}\text { Mental health care referral } \\
\text { and utilization \% (95 \% CI) }\end{array}$ \\
\hline Anxiety & $2.2(1.0-4.8)$ \\
Below symptom threshold \\
Above symptom threshold \\
Impairment not required \\
Mild impairment required & $2.0(0.9-4.1)$ \\
Severe impairment required & $3.1(1.4-6.8)$ \\
Mood & $4.9(1.7-13.7)$ \\
Below symptom threshold & $1.9(0.8-4.2)$ \\
Above symptom threshold & \\
Impairment not required & $44.0(5.0-92.1)$ \\
Mild impairment required & $45.4(4.8-93.2)$ \\
Severe impairment required & $82.0(19.7-98.8)$ \\
ADHD & $1.3(0.6-2.6)$ \\
Below symptom threshold & \\
Above symptom threshold & $26.8(15.3-42.7)$ \\
Impairment not required & $27.8(15.6-44.5)$ \\
Mild impairment required & $37.8(18.6-61.8)$ \\
Severe impairment required & $1.4(0.5-3.4)$ \\
Conduct/oppositional & \\
Below symptom threshold & \\
Above symptom threshold & \\
Impairment not required & Mild impairment required \\
Severe impairment required & $(6.9-29.8)$ \\
\hline
\end{tabular}

Weighted prevalence estimates (95\% confidence intervals) based on Diagnostic Interview Schedule for Children (DISC) interviews in 1,154 selected children to represent rates in the full population-based sample $(N=6,172)$

$A D H D$ attention-deficit/hyperactivity disorder

mild impairment), as indicated by the non-overlapping confidence intervals.

Depending upon the type of disorder, a considerable amount of children who attained symptom thresholds for diagnosis appeared not to show impairment. This was most marked in the case of specific phobia, which, as has been found in other population-based studies [15, $18,20]$, was very common when symptom thresholds alone were considered. However, specific phobia was much less common when mild impairment was required for diagnosis. Conversely, rates of behavior problems decreased considerably only when a more severe degree of impairment was required for diagnosis. The DSM-IV symptom criteria of behavioral diagnoses often require associated impairment at a clinically significant level (e.g., aggression toward other people). Thus, many children who attain symptoms thresholds for diagnosis will also satisfy the impairment criterion [6]. Similarly, for depressive disorders, whose symptoms may be considered to be intrinsically impairing (e.g., markedly diminished interest or pleasure in activities), the requirement of impairment had little or no effect on prevalence [15, 17-21, 28]. One may argue, like Rutter [10] and Spitzer and Wakefield [6], that impairment can be better captured by the modification of symptom criteria. For example, Rutter proposed that "in order to deal with the 'over-diagnosis' of phobias problem, a simple remedy would be to treat avoidance as a symptom rather than an impairment" [10].

One of the few longitudinal studies of child psychiatric disorders beginning in the preschool years showed that the stability of diagnosis was high for behavioral disorders but only moderate for emotional disorders [3]. For example, depressive disorders are substantially more common among adolescents and adults than among young children [29], and adult symptom criteria will less likely identify a valid childhood psychiatric disorder that can be reliably measured using structured diagnostic interviews [30]. Conversely, worries about losing major attachment figures or fear of dogs are substantially more common at younger than at older ages, and rates of separation anxiety disorder or specific phobia diagnosed using developmentally inappropriate criteria may be invalid. Future research is needed to understand the relationships between psychiatric symptoms, different levels of impairment, and diagnosis over time.

Given the structure of the DISC interviews, impairment ratings were obtained only if a certain amount of symptoms was present. It is well established that many children attaining diagnostic thresholds are little impaired, whereas, although not testable here, many children with subthreshold disorders may suffer from associated impairment [20, 28, 31]. Pickles et al. [28] showed that mood and behavioral disorders differ considerably in the extent to which impairment is prognostic. For behavioral disorders, both symptoms and impairment at age 8-16 years were associated with symptoms, diagnosis, and impairment 1.5 years later. For mood disorders, only current symptoms and not impairment scores were associated with future symptoms, diagnosis, and impairment [28]. The relative importance of symptom and impairment criteria for psychiatric classification and clinical decision-making warrants further investigation. We showed that, in addition to symptom criteria, the mild impairment criterion captured virtually all clinically referred cases. However, for most disorders, the severe impairment criterion captured only half of the clinically referred cases.

Although odds ratios for comorbidity increased with the addition of impairment to the diagnostic criteria, confidence intervals were overlapping. Therefore, it cannot 
Table 4 Odds ratios for comorbidity between four DSM-IV diagnostic groupings among 5- to 8-year-old children $(N=6,172)$

\begin{tabular}{|c|c|c|c|c|c|c|c|c|}
\hline \multirow[t]{2}{*}{ Diagnostic groupings } & \multicolumn{2}{|c|}{ Anxiety } & \multicolumn{2}{|c|}{ Mood } & \multicolumn{2}{|c|}{ ADHD } & \multicolumn{2}{|c|}{ Conduct/oppositional } \\
\hline & OR & $(95 \% \mathrm{CI})$ & OR & $(95 \% \mathrm{CI})$ & OR & $(95 \% \mathrm{CI})$ & OR & $(95 \% \mathrm{CI})$ \\
\hline Anxiety & - & - & 1.55 & $(0.36-6.63)$ & 2.62 & $(1.36-5.07)$ & 1.83 & $(0.95-3.53)$ \\
\hline Mood & 2.29 & $(0.51-10.2)$ & - & - & 2.38 & $(0.55-10.2)$ & 2.97 & $(0.66-13.4)$ \\
\hline ADHD & 4.17 & (1.94-8.97) & 2.21 & $(0.50-9.80)$ & - & - & 5.97 & $(3.15-11.3)$ \\
\hline Conduct/oppositional & 2.78 & $(1.28-6.01)$ & 2.80 & $(0.61-12.8)$ & 6.44 & $(3.32-12.5)$ & - & - \\
\hline
\end{tabular}

Weighted odds ratios (ORs) for comorbidity between disorders based on Diagnostic Interview Schedule for Children (DISC) interviews in 1,154 selected children to represent odds ratios in the full population-based sample $(N=6,172)$. CI indicates confidence interval. Upper triangular area values represent odds ratios for disorders without the requirement of impairment and lower triangular values (boldface) represent odds ratios for disorders with mild impairment

$A D H D$ attention-deficit/hyperactivity disorder

be concluded that comorbidity rates differ depending on whether impairment is required or not. The co-occurrence of two or more DSM-IV disorders was common. The current findings concur with those of other investigators [20, 27, 32], indicating that comorbidity is evident among emotional or behavioral disorders, but also across these domains of disorder. Consistent with previous research [15], we observed a marginal gender difference in the prevalence of any psychiatric disorder, with boys outnumbering girls.

Next to the various strengths of our study, including the use of a large prospective population-based cohort, and the collection of data through structured diagnostic interviews, several limitations should be considered. The fact that the current study was conducted in a diverse, urban area is certainly an advantage but does not necessarily imply that findings are easily generalizable to the larger population of children and their families. As in many prospective cohort studies, attrition is a concern. Furthermore, despite being considered an efficient means for estimating prevalence of psychiatric disorders that are relatively uncommon, a twostage sampling design may reduce statistical precision $[25$, 29]. It has been recommended to use multiple reporters in the assessment of outcome when employing a two-stage design [29]. If anything, our use of a single parent informant may have led to an underestimation of the potentially more subtle impairments of children with anxiety disorders versus disruptive disorders. The fact that mental health treatment for children is often initiated by adults, and the possibility that impairment brings symptomatology to the attention of adults [31], may explain the relatively low rates of referral in children with anxiety disorders. However, because of children's young age (5-8 years), children's self-reports of disorders and associated impairment on the DISC would have likely been unreliable [33, 34]. Clearly, a diagnosis based on clinicians' ratings would have been a valuable but costly addition to the structured interviews. Finally, given that assessment waves relied on sending children in a small age range (5-7 years) the same screening questionnaire, we also used the data of those children (41\%) who fell outside the age range for which the questionnaire was designed (age 1.5-5 years). However, tests of internal consistency have indicated that behavioral and emotional problems were also reliably measured in children older than 5 years [35].

In conclusion, findings from this large population-based cohort of 5- to 8-year-old children showed that prevalence was highly dependent upon the criteria used to define impairment. Virtually all children who attained symptom thresholds for a specific disorder, and had been referred to a mental health care professional because of the associated symptoms, also had mild impairment. The requirement of severe impairment criteria significantly increased diagnostic thresholds but for most disorders this definition captured only half of the clinically referred cases. According to the observed mental health care referral and utilization patterns, not only those children with severe levels of impairment, but also those with milder levels should be considered to meet criterion. Findings of this study suggest that if severe impairment is made a diagnostic requirement, many children with psychiatric symptoms and mild impairment seeking mental health care will be undiagnosed and possibly untreated.

Acknowledgments The Generation R Study is conducted by the Erasmus Medical Center Rotterdam in close collaboration with the Erasmus University Rotterdam, the Municipal Health Service Rotterdam area, and the Stichting Trombosedienst and Artsenlaboratorium Rijnmond (STAR). We greatly acknowledge the contribution of participating children and their parents, general practitioners, hospitals, midwives, and pharmacies. We thank all the interviewers who contributed considerable time and expertise to the data in this paper. The general design of the Generation R Study was financially supported by the Erasmus Medical Center Rotterdam, the Erasmus University Rotterdam, and the Netherlands Organization for Health Research and Development (ZonMw, Grant No. 10.000.1003). This study was supported by additional grants from ZonMw (grant No 15.700.1004) and the Sophia Foundation for Scientific Research (SSWO grant number 201310). 
Ethical standards This study was conducted in accordance with the guidelines proposed in the World Medical Association Declaration of Helsinki and was approved by the Medical Ethical Committee of the Erasmus University Medical Center, Rotterdam. Written consent was obtained from all participants.

Conflict of interest Dr. Frank C. Verhulst is a contributing author of the Achenbach System of Empirically Based Assessment (ASEBA), from which he receives remuneration. The other authors declare that they have no conflict of interest.

Open Access This article is distributed under the terms of the Creative Commons Attribution License which permits any use, distribution, and reproduction in any medium, provided the original author(s) and the source are credited.

\section{References}

1. Costello EJ, Angold A, Keeler GP (1999) Adolescent outcomes of childhood disorders: the consequences of severity and impairment. J Am Acad Child Adolesc Psychiatry 38:121-128

2. Esser G, Schmidt MH, Woerner W (1990) Epidemiology and course of psychiatric disorders in school-age childrenresults of a longitudinal study. J Child Psychol Psychiatry 31:243-263

3. Lavigne JV, Arend R, Rosenbaum D, Binns HJ, Christoffel KK, Gibbons RD (1998) Psychiatric disorders with onset in the preschool years: I. Stability of diagnoses. J Am Acad Child Adolesc Psychiatry 37:1246-1254

4. American Psychiatric Association (1994) Diagnostic and Statistical Manual of Mental Disorders, 4th edn. American Psychiatric Association, Washington, DC

5. Ustun B, Kennedy C (2009) What is "functional impairment"? Disentangling disability from clinical significance. World Psychiatry 8:82-85

6. Spitzer RL, Wakefield JC (1999) DSM-IV diagnostic criterion for clinical significance: does it help solve the false positives problem? Am J Psychiatry 156:1856-1864

7. Beals J, Novins DK, Spicer P, Orton HD, Mitchell CM, Baron AE et al (2004) Challenges in operationalizing the DSM-IV clinical significance criterion. Arch Gen Psychiatry 61:1197-1207

8. Regier DA, Kaelber CT, Rae DS, Farmer ME, Knauper B, Kessler RC et al (1998) Limitations of diagnostic criteria and assessment instruments for mental disorders. Implications for research and policy. Arch Gen Psychiatry 55:109-115

9. Wakefield JC (2009) Disability and diagnosis: should role impairment be eliminated from DSM/ICD diagnostic criteria? World Psychiatry 8:87-88

10. Rutter M (2011) Research review: Child psychiatric diagnosis and classification: concepts, findings, challenges and potential. J Child Psychol Psychiatry 52:647-660 (p.656)

11. Wakefield JC, Spitzer RL (2002) Lowered estimates-but of what? Arch Gen Psychiatry 59:129-130

12. American Psychiatric Association (2013) Diagnostic and statistical manual of mental disorders, 5th edn. American Psychiatric Association, Washington, DC

13. Bird HR, Davies M, Fisher P, Narrow WE, Jensen PS, Hoven C et al (2000) How specific is specific impairment? J Am Acad Child Adolesc Psychiatry 39:1182-1189

14. Narrow WE, Rae DS, Robins LN, Regier DA (2002) Revised prevalence estimates of mental disorders in the United States: using a clinical significance criterion to reconcile 2 surveys' estimates. Arch Gen Psychiatry 59:115-123
15. Wichstrom L, Berg-Nielsen TS, Angold A, Egger HL, Solheim E, Sveen TH (2012) Prevalence of psychiatric disorders in preschoolers. J Child Psychol Psychiatry 53:695-705

16. McArdle P, Prosser J, Kolvin I (2004) Prevalence of psychiatric disorder: with and without psychosocial impairment. Eur Child Adolesc Psychiatry 13:347-353

17. Lavigne JV, Lebailly SA, Hopkins J, Gouze KR, Binns HJ (2009) The prevalence of ADHD, ODD, depression, and anxiety in a community sample of 4-year-olds. J Clin Child Adolesc Psychol 38:315-328

18. Carter AS, Wagmiller RJ, Gray SA, McCarthy KJ, Horwitz SM, Briggs-Gowan MJ (2010) Prevalence of DSM-IV disorder in a representative, healthy birth cohort at school entry: sociodemographic risks and social adaptation. J Am Acad Child Adolesc Psychiatry 49:686-698

19. Bird HR, Canino G, Rubio-Stipec M, Gould MS, Ribera J, Sesman M et al (1988) Estimates of the prevalence of childhood maladjustment in a community survey in Puerto Rico. The use of combined measures. Arch Gen Psychiatry 45:1120-1126

20. Simonoff E, Pickles A, Meyer JM, Silberg JL, Maes HH, Loeber $\mathrm{R}$ et al (1997) The virginia twin study of adolescent behavioral development. Influences of age, sex, and impairment on rates of disorder. Arch Gen Psychiatry 54:801-808

21. Verhulst FC, van der Ende J, Ferdinand RF, Kasius MC (1997) The prevalence of DSM-III-R diagnoses in a national sample of Dutch adolescents. Arch Gen Psychiatry 54:329-336

22. Jaddoe VW, van Duijn CM, Franco OH, van der Heijden AJ, van Iizendoorn MH, de Jongste JC et al (2012) The generation R study: design and cohort update 2012. Eur J Epidemiol 27:739-756

23. Achenbach TM, Rescorla LA (2000) Manual for the ASEBA preschool forms and profiles. University of Vermont, Research Center for Children, Youth and Families, Burlington

24. Fisher P, Lucas C (2006) Diagnostic interview schedule for children (DISC-IV) - young child. Columbia University, New York

25. Dunn G, Pickles A, Tansella M, Vazquez-Barquero JL (1999) Two-phase epidemiological surveys in psychiatric research. Br J Psychiatry 174:95-100

26. Julious SA (2004) Using confidence intervals around individual means to assess statistical significance between two means. Pharmaceutical Statistics 3:217-222

27. Ford T, Goodman R, Meltzer H (2003) The British Child and Adolescent Mental Health Survey 1999: the prevalence of DSM-IV disorders. J Am Acad Child Adolesc Psychiatry 42:1203-1211

28. Pickles A, Rowe R, Simonoff E, Foley D, Rutter M, Silberg J (2001) Child psychiatric symptoms and psychosocial impairment: relationship and prognostic significance. Br J Psychiatry 179:230-235

29. Rutter M (1989) Isle of Wight revisited: twenty-five years of child psychiatric epidemiology. J Am Acad Child Adolesc Psychiatry 28:633-653

30. Egger HL, Angold A (2006) Common emotional and behavioral disorders in preschool children: presentation, nosology, and epidemiology. J Child Psychol Psychiatry 47:313-337

31. Angold A, Costello EJ, Farmer EMZ, Burns BJ, Erkanli A (1999) Impaired but undiagnosed. J Am Acad Child Adolesc Psychiatry 38:129-137

32. Verhulst FC, van der Ende J (1993) "Comorbidity" in an epidemiological sample: a longitudinal perspective. J Child Psychol Psychiatry 34:767-783

33. Schwab-Stone M, Fallon T, Briggs M, Crowther B (1994) Reliability of diagnostic reporting for children aged 6-11 years: a testretest study of the Diagnostic Interview Schedule for ChildrenRevised. Am J Psychiatry 151:1048-1054 
34. Shaffer D, Fisher P, Dulcan MK, Davies M, Piacentini J, SchwabStone ME et al (1996) The NIMH Diagnostic Interview Schedule for Children Version 2.3 (DISC-2.3): description, acceptability, prevalence rates, and performance in the MECA Study. methods for the epidemiology of child and adolescent mental disorders study. J Am Acad Child Adolesc Psychiatry 35:865-877
35. Basten M, van der Ende J, Tiemeier H, Althoff RR, Rijlaarsdam J, Jaddoe VW et al (2014) Nonverbal intelligence in young children with dysregulation: the Generation R Study. Eur Child Adolesc Psychiatry 23:1061-1070 